\title{
Ethical Issues in Patient Care in the current COVID-19 Pandemic
}

\author{
Pradeep Narayan ${ }^{1}$, Anjuli Tuladhar Barai ${ }^{2}$, Partha Bhattacharjee ${ }^{2}$, \\ Russell D'Souza ${ }^{3}$, Muralidhar Kanchi ${ }^{4}$ \\ ${ }^{1}$ Department of Cardiac Surgery, NH Rabindranath Tagore International Institute of Cardiac \\ Sciences; Kolkata \\ ${ }^{2}$ Department of Clinical Research, NH Rabindranath Tagore International Institute of Cardiac \\ Sciences; Kolkata, \\ ${ }^{3}$ Head Department of Education, UNESCO Chair in Bioethics, Asia Pacific. \\ ${ }^{4}$ Department of Cardiac Anaesthesia, Narayana Institute of Cardiac Sciences, Bengaluru \\ Corresponding Author: Pradeep Narayan \\ E-mail: pradeepdoc@gmail.com
}

\begin{abstract}
The COVID pandemic continues unabated. Management of COVID patients have brought about huge ethical dilemmas to the fore. There are several concerns and conflicts that afflict the care of these patients. The various ethical issues that have been brought up by the COVID pandemic and measures to mitigate them have been highlighted in the manuscript. Rationing of resources, however clinically justified, is against the ethical principle of Justice which demands equitable distribution of resources and consideration of needs of all patients even while caring for an individual patient. Protecting the patient may be guided by the altruistic principles for the healthcare workers but the price paid by their families is another ethical concern. Diversion of resources to the COVID services compromises care of patients with non-COVID diseases including life threatening conditions like cardiac problems and malignancies. Neglecting these patients once again questions the ethical premise of Justice and equitable distribution of resources. Cardio-Pulmonary Resuscitation and End of life decisions bring about another important ethical concern. The mental anguish of the healthcare workers is another area of concern. The pandemic has also led to an explosion in research. Conduct of these studies and retraction of some major manuscripts highlight further ethical concerns.
\end{abstract}

Key words: COVID-19, ethics, pandemic, patient care

\section{Introduction}

The severe acute respiratory syndrome corona virus 2 (SARS-CoV-2) or Corona-Virus Disease (COVID-19) continues unabated. Countries where the initial peak and trough was reached are now facing newer cases. In the absence of a major breakthrough with Vaccines and "herd immunity" still not being reached it is likely that the pandemic will continue for many more months. This is bound to put a strain on healthcare establishments in terms of infrastructure and manpower perspectives. When resources are limited the distribution of these resources are often influenced by ethical considerations. The imbalances in resources often lead to an emotional disquiet which is difficult to assess objectively but nevertheless are influenced by ethical concerns. While development of evidence-based treatment protocols for COVID -19 patients as well as meeting the critical care needs of non- COVID patients and procedures are essential we also have 
to ensure that the care is provided within ethical principles. Moreover, these ethical principles apply not only to the patients but also to the caregivers and the institution and most importantly to those to who are denied the care owing to the pandemic. The pandemic because of its novelty has brought about a deluge of publications and because of the clinical needs most institutions have relaxed the ethical scrutiny for research. In this review we highlight the main ethical concerns the current pandemic has raised for all the stakeholders.

\section{Ethical Dilemma in Resource Allocation}

Almost $20 \%$ of patients infected with COVID-19 may require critical care support [1]. Mechanical ventilation is the commonest reason for ICU admission [2] and among patients requiring mechanical ventilation the mortality is in excess of $80 \%$ [3]. There are less than one lakh critical care beds in the entire country of which almost $60 \%$ are provided by the private hospitals. Only half of these critical care beds are supported with a ventilator and once again almost two-thirds of these ventilators are provided by the private sector [4].

Irrespective of the source of the critical care beds and the ventilators, the fact remains that these are grossly inadequate to meet the demands imposed by the pandemic. Experience from other centres in the world suggests that almost $20 \%$ of patients infected with SARS-CoV-2 virus may eventually require critical are admission [1]. As a result a strategy has to be developed to appropriately allocate these beds and ventilators to patients. The strategy though clinically relevant imposes serious ethical dilemmas. This is because, the concept of rationing, however clinically justified it may be, is against the ethical principle of Justice which demands equitable distribution of resources and consideration of needs of all patients even while caring for an individual patient [5]. The current pandemic makes this almost impossible in any health care system. Decision to selectively allocate or deny ventilators and resources pose an ethical dilemma. Denying elderly people the care they require at this stage of the their life reeks of "ageism" whereas denying a younger more fit person a ventilator would not only nip a young life in bud but would also indirectly may ruin an entire family that may be dependent on him. The choice is between a rock and a hard place. This dilemma has been witnessed by several western countries too and in general the consensus was to dedicate the scarce resource of critical care beds and ventilators to those who have the highest chance of surviving. This is very similar to the medical and ethical principles used during previous pandemics and other disaster management protocols [6]. When care is provided based on rationing it is inevitable to draw a backlash from society. It is therefore important to develop an ethical framework that reflects the expectations and priorities of the society with respect to resource allocation [7]. It has been pointed out that in a pandemic the focus should shift from a patient-centred provision of healthcare, to a community-centred model of health provision [8].

Prompted by the situation in Lombardy, Italy, the Italian College of Anaesthesia, Analgesia, Resuscitation, and Intensive Care (SIAARTI) recommended a combination of "clinical reasonableness" as well as a "soft utilitarian" approach to deal with the issue of limited resources and dilemmas in resource allocation [9]. The rationality of clinical reasonableness often failed to justify the emotional dilemma and these decisions prompted several physicians to seek ethical counsel.

\section{The price paid by non-COVID patients}

Another important problem and ethical issue brought out by the pandemic is the treatment of patients who do not have COVID. In order to deal with COVID patients there has been significant restructuring of services across the globe with different bodies providing recommendations as to how this reorganisation of services can be carried out [10-12]. However, the focus of these measures taken is the COVID patient and the non-COVD patients have unfortunately been neglected to a large extent.

Cardiac surgery for instance has seen a massive reduction in performing life-saving procedures. The hospitals are dictated by tiers they fall in and the treatment that can be offered to non-COVID patients is influenced by the COVID workload. For instance, in cardiac surgery, hospitals have been classified into 4 tiers with a proposed reduction in operative capacity. Tier $1(0-30 \%$ inpatient COVID-19 Load, mild reduction in operative capacity); Tier 2 (30-60\% inpatient COVID-19 Load, 
moderate reduction in operative capacity); Tier 3 (60-80\% inpatient COVID-19 Load, severe reduction in operative capacity) and Tier 4 (>80\% inpatient COVID Load, minimal operative capacity) [11].

Similarly, Primary Percutaneous Coronary Interventions (PPCI) which is the mainstay of treatment of ST-elevation myocardial infarction (STEMI) has been affected severely. The duration between occurrence of pain and the intervention being carried out is the key to successful treatment. It has been seen during the pandemic the number of STEMI cases treated were significantly reduced. It is unlikely to be due to a sudden decrease in the occurrence of STEMI but is more likely to be an outcome of redistribution of resources. Further credence to this theory is found by the fact that even when patients were diagnosed with STEMI there was a significant delay between the onset of symptoms and PPCI [13].

Apart from cardiac patients, other specialities like Orthopaedics, Gastroenterology, Dermatology, Urology and many other chronic conditions have all seen significant drop in patient numbers presenting to the hospitals [14]. Endoscopies were reduced dramatically all across the globe due to concerns over it being an AGP. Some countries reported a 90\% reduction in the endoscopy rates [15]. Screening tests for important conditions like diabetes and dyslipidemia have also witnessed a dramatic dip. While undoubtedly these screening tests are less urgent than cancer and other emergencies the cumulative risk of undiagnosed diabetes and dyslipidemia tends to be much higher. If nothing it mirrors the general neglect of non-COVID conditions which has led to an unaccountable suffering. Some of these neglected areas like screening for diabetes and dyslipidemia may have long term impact and may present as significant economic and health burden in future [16]. Once again, these measures question the ethical premise of Justice and equitable distribution of resources.

\section{Cancer services and the conflict between Beneficence and Maleficence}

Cancer patients are a very vulnerable cohort as concomitant infection with COVID-19 carries a high risk of mortality. However, delays in diagnosis, surveillance or treatment all have serious implications towards the overall prognosis [5]. In the wake of the pandemic, several changes have been brought about regarding screening and treatment modalities. Regimens for surgery, chemotherapy, radiotherapy have all been altered.

Re-allocation of resources has led to reduction or in some instances, complete suspension of cancer screening services. This has serious implications. Urgent referrals of cancer patients have seen a drastic reduction in many countries that invariably leads to lesser number of cancers being diagnosed [17]. Chemotherapy protocols are being modified to minimize the frequency of visits and degree of immunosuppression. Maintenance therapy is not recommended in order to reduce hospital visits. The role of radiotherapy has expanded with COVID-19, as radiation therapy is being used to replace or delay surgery. For instance, a radiotherapy has been advised to delay surgery in patients with rectal cancer [18]. This may have implications on the prognosis and also puts extra demand on radiotherapy services eventually leading to selective allocation.

Surgical care of cancer patients has suffered during the pandemic. Patients previously treated with or designated for surgery are being treated with other form of therapies [19]. With the focus mainly on emergency care almost all the countries, took measures to delay or defer non-emergency surgeries. The delay in cancer surgery has potential for serious harm. Delaying certain cancer surgeries by few weeks can result in an increase in tumour size and may even convert an operable case into an inoperable one changing the prognosis completely [20]. For example, certain patients with head and neck tumours have excellent prognosis after surgical resection and denying them the surgery and the cure in face of the pandemic is against ethical principles [21].

The recommendations of the European Society for Medical Oncology to classify certain cancer as low or medium priority for follow-up may have serious implications for most cancers in terms of depending on risk of disease and complications. Also, face to face consultations have been replaced with online consultations. Breaking bad news or stating the poor prognosis of the cancer is difficult at best of times. To do so online is going to be even more challenging [10]. Studies have shown that there was significant concern among patients with cancer both about acquiring COVID as a 
nosocomial infection as well as with regards to disruption of their standard prescribed care due to by COVID-19 [22].

\section{Protecting the patient at the cost of the family of the Healthcare worker- another ethical concern}

One ethical dilemma that is rarely talked about is the conflict between serving the population at large and protecting one's family. The dedication and devotion of healthcare workers (HCW) is a prime example of altruism [23]. However, the HCW's working in the critical care areas designated for patients infected with COVID-19 are at risk of acquiring the infection. Not only that they are at risk of passing it on to their family members especially if it consists of elderly parents and young children. Various HCW's are resorting to stay away from elderly parents and sending children away to grandparents to protect them. Others choose to stay together and live under constant fear of infecting their loved ones. It is not just the health of the family of the HCW at risk but also there are significant psychological and financial factors that come into play making this an extremely difficult dilemma to address. To make it worse this is not even discussed or thought about either by the institution they work for or by the society at large. Most health care institutions do not provide automatic insurance cover for the family of the HCW. Thus, purchasing the right insurance that covers the elderly family members is the onus of the HCW and in the current scenario is extremely difficult and expensive. This coupled with the fact that most HCW's are receiving a reduced pay from the healthcare establishments make this ironical. It is not at all surprising that the HCW's are under psychological distress and burnout [24].

This is another area where the ethical responsibility of the healthcare establishment comes into focus. There is no doubt that hospitals should consider proper residential and refreshments for those directly involved in critical care services looking after COVID patients. The bigger question is should the healthcare establishment go any further and provide free healthcare if the HCW or any of the family members of the HCW contracts COVID? On surface it may be tempting to suggest that they should. However, this is also questionable. How does the hospital establish that the family member was staying with the HCW? How can it be assessed that the family member was infected by the HCW? While these are difficult questions and the answers will vary from region to region and hospital to hospital however at the bare minimum the hospital should think of these ethical issues. The HCW should be given a choice to stay in the hospital during the period of duty and longer if they prefer to stay away from their family for a while to ensure they are not infected [25]. It is not just the frontline workers, like doctors and nurses but also the support staff like the receptionists, managers, housekeeping, that face this dilemma. HCWs, in other areas like laundry and facilities, often feel left out and disillusioned [26].

Several measures can be taken to deal with the concerns and dilemmas of the HCW. Communication and leadership and empathy are the key to respond adequately to the concerns of the HCWs. Besides, reduction of noncritical work, information on managing stress, sessions to provide psychological support either as a group or individually will help [27-28].

\section{Patient's Family- Another un-quantified ethical concern}

Most families in current times are nuclear and once a person is diagnosed with COVID and needs admission in a critical care area it throws a multitude of issues on the family front. Emotional stress brought out by the uncertainties of the prognosis is further magnified by the added anguish of physical separation [29]. Financial constraints and stress on children are other issues that are major concerns.

The emotional trauma to the patient and the families during this pandemic is of unthinkable proportions. The inability of family members to stay next to their loved ones during their last moments generates emotions that cannot be recorded in words. Having the person, with whom you have shared most of your life, next to you, when you are breathing your last, is the best palliation. Denying the basic need to bid farewell to loved ones results in depression, and guilt in the surviving family members that can last a lifetime. The patients themselves have a social craving and a primordial need to see their families [30]. Despite the HCW's doing their best the critical 
care environment during current pandemic is greatly dehumanized with members of the caring team covered in their PPE [31].

Dying from COVID-19 in isolation has existential as well as social consequences for both patients and families [32]. In many countries like India the funeral rites of a COVID patient is performed by the state with the family not having access to the dead body. In fact in some states, they are not even allowed to the cremation site. Funeral rituals are fundamentally ingrained in our culture and rites are important to signify the end of life. Several cultural and religious beliefs are inherent to the last rites of a person and serve as a closure for the families. Religious rituals are considered as the final dignity that can be offered to the departed and is deeply entrenched in our psycho-social and religious and cultural beliefs. The pandemic and the preventive measures taken deny this basic need of families and is a major ethical concern [31]. Changes should be instituted to allow family members participate in the last rituals of the deceased patient in a safe way. Other methods to maintain communication like video calls and supervised short visits with adequate precautions should be considered.

\section{Cardio-Pulmonary Resuscitation and End of life decisions}

Cardiopulmonary resuscitation poses another important ethical dilemma. At one end there is a very short window of opportunity to resuscitate a patient with COVID-19 who has arrested on the other hand there is a highly increase risk of contamination to the HCW.

Both chest compressions and endotracheal intubation of bag and mask ventilation are aerosolgenerating procedures (AGPs). As a result, cardiopulmonary resuscitation has a huge potential to generate aerosols [33]. HCW's are used to instituting immediate chest compression and airway control algorithms but are now confronted with the dilemma between wearing personal protective equipment and instituting immediate CPR protocols. The current recommendations made by the European Resuscitation Council COVID-19 guidelines suggest that a minimum a FFP3 mask (FFP2 or N95 if FFP3 not available), eye and face protection, long-sleeved gown, and gloves should be donned before undertaking CPR. However, DC cardioversion can be attempted prior to gaining airway control and chest compressions [34].

More importantly however hard it may be a careful assessment has to be made to identify those where CPR would be inappropriate [34]. Patients with severe COVID-19 respiratory failure and multiple organ failure who undergo a cardiac arrest may not be candidates for CPR. While the decision for not attempting CPR used to be taken in the past too they have assumed far greater importance in the current pandemic and have significant emotional and ethical challenges for the health care workers as well as the family members.

Some of the other measures that can be taken to mitigate the ethical concerns to a certain extent regarding CPR include using mechanical compression devices in COVID-19 as well as performing CPR with the patient in the prone position. These measures can potentially limit the number of required people during the CPR and also limit viral exposure [35-36].

Some patients are because of age and other co-morbid conditions may have a much poorer outlook. In these patients initiating mechanical ventilation may not prove to be of much use. While the patient is rapidly deteriorating, with family members not around the doctor invariably decided on the best possible option. However, that option may not be the preferred option by the patient who at the time of decision making may not be in a state to exercise or inform his wish. The fact that end of life decisions and care even in a pandemic is primarily a patients choice it is important that decision-making is shared and communication with the patient and the family in advance should remain central to clinical practice [37].

It has been witnessed that end of life discussions with COVID patients are significantly lower compared to pre-COVID times. Also, in only 1 in 4 cases a relative was present at the time of death in the hospitals [32]. The role of advance directives thus has assumed greater importance especially in the Western world.

Virtual intensive care unit (vICU) is another novel measure that has been taken to allow family for patients with COVID-19. Patients are able to virtually see and hear their family members which helped with the mental strength to fight through the crisis. It also serves as a boon for the families who have expressed gratitude for the ability to see and speak with their loved ones [38]. 
Tele-palliative medicine is a similar measure implemented to allow family members the ability to interact with their loved ones during the end of life. It helps with care and end of life discussions, provides contact and closure for both patients and their families [39].

\section{Vulnerabilities of Health Care Workers}

The pandemic has brought about not only health concerns but also a psychosocial burden on the healthcare workers. Healthcare workers are at high risk of acquiring nosocomial acquisition of SARS-CoV-2 and are also predisposed to burnout, anxiety, fear of transmission, depression, substance abuse and strategies that aim to prevent and treat vicarious traumatization in medical staff have to be developed [40].

\section{Ethics and COVID research}

It is important to engage in research in the current pandemic as important answers regarding the treatment and prevention can only be obtained through meticulous study of the infected patients. While the studies may or may not benefit the participants, it can be deemed to serve the population at large. In order to facilitate important research in an expeditious fashion many health authorities have adopted fast track policy for ethics approval in COVID research [41]. Majority of journals are also relaxing the requirements of peer review in order to provide important scientific information in the public domain in a timely fashion. However, one of the unprecedented fallout of the COVID pandemic and encouraging research is the perverse explosion in the number of publications using COVID patients as "subjects' of research.

Broadly there are two important ethical concerns with regards to research in COVID patients. Firstly, to ensure that the basic principles of research ethics are adhered to and secondly that in the mad rush to publish, the quality and sanctity of the research is not compromised. The age old adage of "Bad research is Bad Ethics" has to be adhered to. With regards to the first concern there are some thought provoking data that has come to light. A significant proportion of studies are recruiting or reporting patient data include patients who are more than 65 years of age and one in six participants in these studies are children [42].

A cursory search on PubMed alone using the search term "COVID" resulted in 58,740 results. This is an absurd amount of publication generated in less than 9 months that translates into more than 6500 publications in PubMed indexed journals every month. Most of these publications have been rushed through and there are concerns over the quality of peer review in many of those. A recent publication on the subject confirmed that almost 4000 papers were made available on preprint servers, a number that is only likely to increase. By $31^{\text {st }}$ of July 2020,33 manuscripts had been retracted or withdrawn or concerns had been raised regarding them. $12 \%$ (4 out of 33) were retracted in high profile journals like Lancet and New England Journal of Medicine [43-46]. Of the studies where an issue was flagged there were six which were due to consent issues and 3 had research ethics committee implications. These studies may just be the tip of the ice-berg only identified because of being published in journals with higher impact factor and more analytical readership.

One of the most obvious and practical concerns over research in COVID patients centres around informed consent. As researchers are usually not allowed in the restricted COVID designated zones the sanctity of informed consent is realistically under question. Delegating the consent responsibility to the frontline health workers is now recommended as one of the strategies for consenting [47].

However, concerns remain over the efficiency and due diligence exercised by the overworked frontline worker in obtaining the consent. Obtaining informed consent through alternative means like mobile apps and tele-consenting makes good reading but the use is limited to very limited healthcare sectors and remains a very theoretical proposition in most institutions.

This is a very coarse ethical issue and much more subtle concerns regarding the mental well being of the participant or the legally acceptable representative (LAR) is not often accounted for. A person in critical care fighting for his life in a dehumanized environment confronted with the prospect of being a research participant is bound to have significant mental issues. Similar implications exist for the LAR consenting on behalf of the patient and are perhaps not accounted 
for. It is recommended that the impacts of COVID-19 on mental well being of participants and relatives should be considered even if these studies are not designed to evaluate mental health issues [48]. Ethical concerns also exist with conduct of RCTs in COVID patients. It is inevitable that participants in the control arm would receive the best alternative medical or supportive care and may be denied the opportunity to have the investigational drug or vaccine and a favourable outcome [49]. It is obvious that research in patient with COVID, especially those in critical care present a huge challenge from the ethical perspective. In order to mitigate these concerns, it is imperative for researchers to adhere to guidance provided by national bodies [50]. Besides, the WHO has listed ethical standards that is expected to be followed by researchers during this pandemic [51].

Answers for ethical dilemmas are never right or wrong, but are just difficult ones. Protecting patients with COVID infection as well those who have other chronic illnesses is equally important and in line with the ethical principles of distributive justice and beneficence and non-maleficence. The respect of the wishes of the patient and their families has to be respected and should be guided by respect for autonomy. In doing so it is important to protect the healthcare workers to safeguard the principle of fairness and human rights.

\section{REFERENCES}

1. Guan W, Ni Z, Hu Y, Liang W, Ou C, He J. Clinical Characteristics of Coronavirus Disease 2019 in China. N Engl J Med 2020;Feb 28 (Online ahead of print).

2. Richardson S, Hirsch JS, Narasimhan M, Crawford JM, McGinn T, Davidson KW et al. Presenting Characteristics, Comorbidities, and Outcomes Among 5700 Patients Hospitalized With COVID19 in the New York City Area. JAMA 2020 Apr 22;(online ahead of print).

3. Arentz M, Yim E, Klaff L, Lokhandwala S, Riedo FX, Chong M, et al. Characteristics and Outcomes of 21 Critically Ill Patients With COVID-19 in Washington State. JAMA 2020;323(4):1612-4.

4. Kapoor G, Sriram A, Joshi J, Nandi A, Laxminarayan R. COVID-19 in India: State-wise estimates of current hospital beds, intensive care unit (ICU) beds and ventilators. Center for Disease, Dynamics, Economics, and Policy. Retrieved from https://cddep. org/wpcontent/uploads/2020/04/State-wise-estimates-of-current-beds-and-ventilators_24Apr2020. pdf. 2020 Apr 20.

5. Harkin DW. Ethics for surgeons during the COVID-19 pandemic, review article. Ann Med Surg 2020;55:316-9.

6. Daugherty Biddison EL, Faden R, Gwon HS, Mareiniss DP, Regenberg AC, Schoch-Spana M, et al. Too Many Patients...A Framework to Guide Statewide Allocation of Scarce Mechanical Ventilation During Disasters. Chest 2019;155(4):848-54.

7. Rosenbaum L. Facing COVID-19 in Italy - Ethics, Logistics, and Therapeutics on the Epidemic's Front Line. N Engl J Med 2020;382(20):1873-5.

8. Mirco N, Andrea C, Angelo G, Pietro B, Federico L, Pisano M. At the Epicenter of the COVID19 Pandemic and Humanitarian Crises in Italy: Changing Perspectives on Preparation and Mitigation. NEJM Catalysts Innovative Health Care Delivery 2020;Mar 21.

9. Vergano M, Bertolini G, Giannini A, Gristina GR, Livigni S, Mistraletti G, RICCIONI L, PETRINI F. SIAARTI recommendations for the allocation of intensive care treatments in exceptional, resource-limited circumstances. Minerva Anestesiol 2020;Apr 3:1-8.

10. Richards M, Anderson M, Carter P, Ebert BL, Mossialos E. The impact of the COVID-19 pandemic on cancer care. Nat Cancer 2020; May 20;1-3.

11. Haft JW, Atluri P, Alawadi G, Engelman D, Grant MC, Hassan A, et al. Adult cardiac surgery during the COVID-19 Pandemic: A Tiered Patient Triage Guidance Statement. Ann Thorac Surg 2020;Apr 10 (online ahead of print).

12. Hussain A, Balmforth D, Yates M, Lopez-Marco A, Rathwell C, Lambourne J, et al. The Pan London Emergency Cardiac Surgery service: Coordinating a response to the COVID-19 pandemic. J Card Surg 2020;35(7):1563-9.

13. Çinier G, Hayıroğlu M, Pay L, Yumurtaş A, Tezen O, Parsova KE, et al. Effect of the COVID-19 pandemic on access to primary percutaneous coronary intervention for ST-segment elevation myocardial infarction. Turk Kardiyol Dernegi Arsivi Turk Kardiyol Derneginin Yayin Organidir. 2020;48(7):640-5.

14. Mauro V, Lorenzo M, Paolo C, Sergio H. Treat all COVID 19-positive patients, but do not forget 
those negative with chronic diseases. Intern Emerg Med 2020;Jun 9;1-4.

15. NED - National Endoscopy Database.Available from: https://nedpilot.thejag.org.uk/Default.aspx?ReturnUrl=\%2f

16. Wright A, Salazar A, Mirica M, Volk LA, Schiff GD. The Invisible Epidemic: Neglected Chronic Disease Management During COVID-19. J Gen Intern Med 2020;Jul 14;1-2.

17. april2020_cruk_hsc_submission_COVID_cancer_final_public.pdf [Internet]. [cited 2020 Oct 10]. Available from: https://www.cancerresearchuk.org/sites/default/files/april2020_cruk_hsc_submission_COVID_ cancer_final_public.pdf

18. Marijnen C a. M, Peters FP, Rödel C, Bujko K, Haustermans K, Fokas E, et al. International expert consensus statement regarding radiotherapy treatment options for rectal cancer during the COVID 19 pandemic. Radiother Oncol 2020;148:213-5.

19. Rosenbaum L. The Untold Toll - The Pandemic's Effects on Patients without COVID-19. N Eng1 J Med 2020;382(24):2368-71.

20. COVID19 Subcommittee of the O.R. Executive Committee at Memorial Sloan Kettering. Cancer Surgery and COVID19. Ann Surg Oncol 2020;27(6):1713-6.

21. Gordin EA, Day A, Stankova L, Heitman E, Sadler J. Care in the time of coronavirus: Ethical considerations in head and neck oncology. Head Neck 2020; May 21 (online ahead of print).

22. Guven DC, Sahin TK, Aktepe OH, Yildirim HC, Aksoy S, Kilickap S. Perspectives, Knowledge, and Fears of Cancer Patients About COVID-19. Front Oncol 2020;Aug 28 (online ahead of print).

23. Wang H, Liu Y, Hu K, Zhang M, Du M, Huang H, et al. Healthcare workers' stress when caring for COVID-19 patients: An altruistic perspective. Nurs Ethics 2020;27(7):1490-500.

24. Dobson H, Malpas CB, Burrell AJ, Gurvich C, Chen L, Kulkarni J, et al. Burnout and psychological distress amongst Australian healthcare workers during the COVID-19 pandemic. Australas Psychiatry Bull R Aust N Z Coll Psychiatr 2020;1039856220965045.

25. Menon V, Padhy SK. Ethical dilemmas faced by health care workers during COVID-19 pandemic: Issues, implications and suggestions. Asian J Psychiatry 2020;51:102116.

26. Wu AW, Connors C, Everly GS. COVID-19: Peer Support and Crisis Communication Strategies to Promote Institutional Resilience. Ann Intern Med 2020;172(12):822-3.

27. Adams JG, Walls RM. Supporting the Health Care Workforce During the COVID-19 Global Epidemic. JAMA;323(15):1439-40.

28. Dewey C, Hingle S, Goelz E, Linzer M. Supporting Clinicians During the COVID-19 Pandemic. Ann Intern Med 2020;172(11):752-3.

29. Hart JL, Turnbull AE, Oppenheim IM, Courtright KR. Family-Centered Care During the COVID19 Era. J Pain Symptom Manage 2020;60(2):e93-7.

30. The need to connect: Acute social isolation causes neural craving responses similar to hunger I bioRxiv [Internet]. [cited 2020 Oct 11]. Available from: https://www.biorxiv.org/content/10.1101/2020.03.25.006643v1

31. Guessoum SB, Moro MR, Mallet J. The COVID-19 Pandemic: Do Not Turn the Health Crisis Into a Humanity Crisis. Prim Care Companion CNS Disord 2020;22(4).

32. Strang P, Bergström J, Martinsson L, Lundström S. Dying From COVID-19: Loneliness, End-ofLife Discussions, and Support for Patients and Their Families in Nursing Homes and Hospitals. A National Register Study. J Pain Symptom Manage 2020;60(4):e2-13.

33. Perkins GD, Morley PT, Nolan JP, Soar J, Berg K, Olasveengen T, et al. International Liaison Committee on Resuscitation: COVID-19 consensus on science, treatment recommendations and task force insights. Resuscitation 2020;151:145-7.

34. Nolan JP, Monsieurs KG, Bossaert L, Böttiger BW, Greif R, Lott C, et al. European Resuscitation Council COVID-19 guidelines executive summary. Resuscitation 2020;153:45-55.

35. Cheruku S, Dave S, Goff K, Park C, Ebeling C, Cohen L, et al. Cardiopulmonary Resuscitation in Intensive Care Unit Patients With Coronavirus Disease 2019. J Cardiothorac Vasc Anesth. 2020;34(10):2595-603.

36. Mazer SP, Weisfeldt M, Bai D, Cardinale C, Arora R, Ma C, et al. Reverse CPR: a pilot study of CPR in the prone position. Resuscitation 2003;57(3):279-85.

37. Simpson N, Milnes S, Steinfort D. Don't forget shared decision-making in the COVID-19 crisis. Intern Med J 2020;50(6):761-3.

38. Dhala A, Sasangohar F, Kash B, Ahmadi N, Masud F. Rapid Implementation and Innovative Applications of a Virtual Intensive Care Unit During the COVID-19 Pandemic: Case Study. J Med Internet Res 2020;22(9):e20143.

39. Flores S, Abrukin L, Jiang L, Titone L, Firew T, Lee J, et al. Novel Use of Telepalliative Care in a New York City Emergency Department During the COVID-19 Pandemic. J Emerg Med 2020;Aug 
4 (online ahead of print).

40. Li Z, Ge J, Yang M, Feng J, Qiao M, Jiang R, et al. Vicarious traumatization in the general public, members, and non-members of medical teams aiding in COVID-19 control. Brain Behav Immun. 2020;Mar 10 (online ahead of print).

41. COVID-19 research [Internet]. Health Research Authority. [cited 2020 Oct 12]. Available from: /COVID-19-research/

42. Bramstedt KA. The carnage of substandard research during the COVID-19 pandemic: a call for quality. J Med Ethics 2020; Oct 1 (online ahead of print).

43. The Editors Of The Lancet Global Health. Retraction-Chinese medical staff request international medical assistance in fighting against COVID-19. Lancet Glob Health. 2020;8(8):e995.

44. Mehra MR, Ruschitzka F, Patel AN. Retraction-Hydroxychloroquine or chloroquine with or without a macrolide for treatment of COVID-19: a multinational registry analysis. Lancet Lond Engl 2020;395(10240):1820.

45. Mehra MR, Desai SS, Kuy S, Henry TD, Patel AN. Retraction: Cardiovascular Disease, Drug Therapy, and Mortality in COVID-19. N Engl J Med 2020;382(26):2582.

46. Fouque D, Nouvier M. Retraction: First viral replication of COVID-19 identified in the peritoneal dialysis fluid. Bull Dial À Domic [Internet]. 2020 Apr 20 [cited 2020 Oct 12];3(1). Available from: https://bdd.rdplf.org/index.php/bdd/article/view/54713

47. Kumar NK, Muthuswamy V. Fostering ethical biomedical and health research in India during the COVID-19 pandemic. Res Ethics 2020;;16(3-4):1-10.

48. Townsend E, Nielsen E, Allister R, Cassidy SA. Key ethical questions for research during the COVID-19 pandemic. Lancet Psychiatry 2020;7(5):381-3.

49. Meagher KM, Cummins NW, Bharucha AE, Badley AD, Chlan LL, Wright RS. COVID-19 Ethics and Research. Mayo Clin Proc 2020;95(6):1119-23.

50. Thakur DK. National Guidelines for Ethics Committees reviewing biomedical \& research.

51. liverecovery-save-of-ethical-standards-for-research-during-public-health-emergencies.pdf

[Internet]. [cited 2020 Oct 12]. Available from: https://www.who.int/blueprint/prioritydiseases/key-action/liverecovery-save-of-ethical-standards-for-research-during-public-healthemergencies.pdf?ua $=1$

Acknowledgements: Nil

Funding: Nil

Conflict of interest: Nil 